\title{
Using a Probabilistic Class-Based Lexicon for Lexical Ambiguity Resolution
}

\author{
Detlef Prescher and Stefan Riezler and Mats Rooth \\ Institut für Maschinelle Sprachverarbeitung \\ Universität Stuttgart, Germany
}

\begin{abstract}
This paper presents the use of probabilistic class-based lexica for disambiguation in targetword selection. Our method employs minimal but precise contextual information for disambiguation. That is, only information provided by the target-verb, enriched by the condensed information of a probabilistic class-based lexicon, is used. Induction of classes and fine-tuning to verbal arguments is done in an unsupervised manner by EM-based clustering techniques. The method shows promising results in an evaluation on real-world translations.
\end{abstract}

\section{Introduction}

Disambiguation of lexical ambiguities in naturally occuring free text is considered a hard task for computational linguistics. For instance, word sense disambiguation is concerned with the problem of assigning sense labels to occurrences of an ambiguous word. Resolving such ambiguities is useful in constraining semantic interpretation. A related task is target-word disambiguation in machine translation. Here a decision has to be made which of a set of alternative target-language words is the most appropriate translation of a source-language word. A solution to this disambiguation problem is directly applicable in a machine translation system which is able to propose the translation alternatives. A further problem is the resolution of attachment ambiguities in syntactic parsing. Here the decision of verb versus argument attachment of noun phrases, or the choice for verb phrase versus noun phrase attachment of prepositional phrases can build upon a resolution of the related lexical ambiguities.

Statistical approaches have been applied successfully to these problems. The great advantage of statistical methods over symbolic-linguistic methods has been deemed to be their effective exploitation of minimal linguistic knowledge. However, the best performing statistical approaches to lexical ambiguity resolution themselves rely on complex information sources such as "lemmas, inflected forms, parts of speech and arbitrary word classes [... ] local and distant collocations, trigram sequences, and predicate argument association" (Yarowsky (1995), p. 190) or large context-windows up to 1000 neighboring words (Schütze, 1992). Unfortunately, in many applications such information is not readily available. For instance, in incremental machine translation, it may be desirable to decide for the most probable translation of the arguments of a verb with only the translation of the verb as information source but no large window of surrounding translations available. In parsing, the attachment of a nominal head may have to be resolved with only information about the semantic roles of the verb but no other predicate argument associations at hand.

The aim of this paper is to use only minimal, but yet precise information for lexical ambiguity resolution. We will show that good results are obtainable by employing a simple and natural look-up in a probabilistic class-labeled lexicon for disambiguation. The lexicon provides a probability distribution on semantic selection-classes labeling the slots of verbal subcategorization frames. Induction of distributions on frames and class-labels is accomplished in an unsupervised manner by applying the EM algorithm. Disambiguation then is done by a simple look-up in the probabilistic lexicon. We restrict our attention to a definition of senses as alternative translations of source-words. Our approach provides a very natural solution for such a target-language disambiguation task - look for the most frequent target-noun whose semantics fits best with the 


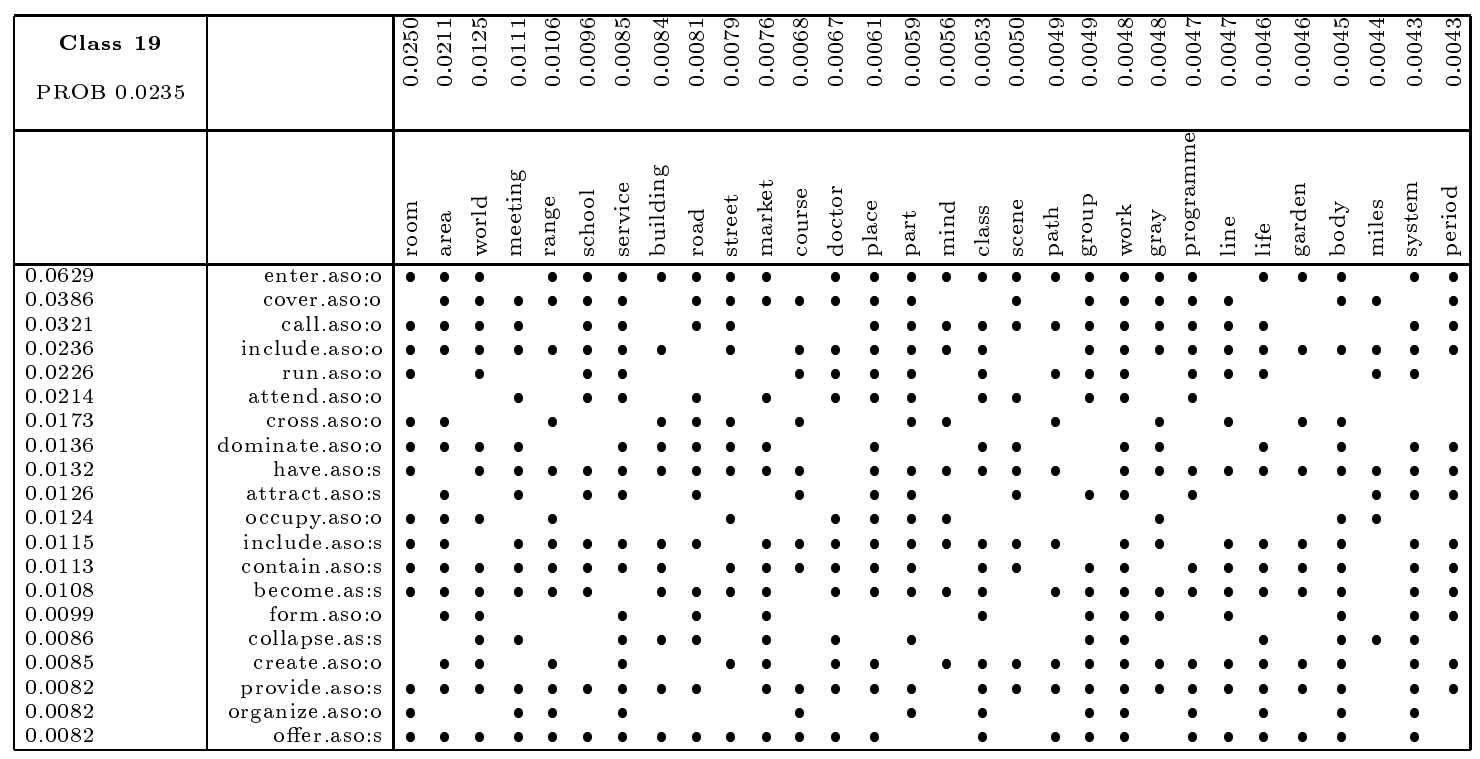

Figure 1: Class 19: "locative action". At the top are listed the 20 most probable nouns in the $p_{L C}(n \mid 19)$ distribution and their probabilities, and at left are the 30 most probable verbs in the $p_{L C}(v \mid 19)$ distribution. 19 is the class index. Those verb-noun pairs which were seen in the training data appear with a dot in the class matrix. Verbs with suffix .as $: s$ indicate the subject slot of an active intransitive. Similarily .aso $: s$ denotes the subject slot of an active transitive, and .aso : $o$ denotes the object slot of an active transitive.

semantics required by the target-verb. We evaluated this simple method on a large number of real-world translations and got results comparable to related approaches such as that of Dagan and Itai (1994) where much more selectional information is used.

\section{Lexicon Induction via EM-Based Clustering}

\subsection{EM-Based Clustering}

For clustering, we used the method described in Rooth et al. (1999). There classes are derived from distributional data - a sample of pairs of verbs and nouns, gathered by parsing an unannotated corpus and extracting the fillers of grammatical relations. The semantically smoothed probability of a pair $(v, n)$ is calculated in a latent class (LC) model as $p_{L C}(v, n)=$ $\sum_{c \in C} p_{L C}(c, v, n)$. The joint distribution is defined by $p_{L C}(c, v, n)=p_{L C}(c) p_{L C}(v \mid c) p_{L C}(n \mid c)$. By construction, conditioning of $v$ and $n$ on each other is solely made through the classes $c$. The parameters $p_{L C}(c), p_{L C}(v \mid c), p_{L C}(n \mid c)$ are estimated by a particularily simple version of the EM algorithm for context-free models.
Input to our clustering algorithm was a training corpus of $1,178,698$ tokens (608,850 types) of verb-noun pairs participating in the grammatical relations of intransitive and transitive verbs and their subject- and object-fillers. Fig. 1 shows an induced class from a model with 35 classes. Induced classes often have a basis in lexical semantics; class 19 can be interpreted as locative, involving location nouns "room", "area", and "world" and verbs as "enter" and "cross".

\subsection{Probabilistic Labeling with Latent Classes using EM-estimation}

To induce latent classes for the object slot of a fixed transitive verb $v$, another statistical inference step was performed. Given a latent class model $p_{L C}(\cdot)$ for verb-noun pairs, and a sample $n_{1}, \ldots, n_{M}$ of objects for a fixed transitive verb, we calculate the probability of an arbitrary object noun $n \in N$ by $p(n)=\sum_{c \in C} p(c, n)=$ $\sum_{c \in C} p(c) p_{L C}(n \mid c)$. This fine-tuning of the class parameters $p(c)$ to the sample of objects for a fixed verb is formalized again as a simple instance of the EM algorithm. In an experiment with English data, we used a clustering model with 35 classes. From the maximum probabil- 
ity parses derived for the British National Corpus with the head-lexicalized parser of Carroll and Rooth (1998), we extracted frequency tables for transitive verb-noun pairs. These tables were used to induce a small class-labeled lexicon (336 verbs).

\begin{tabular}{|lr|}
\hline cross.aso:o 19 & 0.692 \\
\hline \hline mind & 74.2 \\
road & 30.3 \\
line & 28.1 \\
bridge & 27.5 \\
room & 20.5 \\
border & 17.8 \\
boundary & 16.2 \\
river & 14.6 \\
street & 11.5 \\
atlantic & 9.9 \\
\hline
\end{tabular}$\quad$\begin{tabular}{ll} 
mobilize.aso:o 6 & 0.386 \\
\hline
\end{tabular}

Figure 2: Estimated frequencies of the objects of the transitive verbs cross and mobilize

Fig. 2 shows the topmost parts of the lexical entries for the transitive verbs cross and mobilize. Class 19 is the most probable class-label for the object-slot of cross (probability 0.692); the objects of mobilize belong with probability 0.386 to class 16 , which is the most probable class for this slot. Fig. 2 shows for each verb the ten nouns $n$ with highest estimated frequencies $f_{c}(n)=f(n) p(c \mid n)$, where $f(n)$ is the frequency of $n$ in the sample $n_{1}, \ldots, n_{M}$. For example, the frequency of seeing mind as object of cross is estimated as 74.2 times, and the most frequent object of mobilize is estimated to be force.

\section{Disambiguation with Probabilistic Cluster-Based Lexicons}

In the following, we will describe the simple and natural lexicon look-up mechanism which is employed in our disambiguation approach. Consider Fig. 3 which shows two bilingual sentences taken from our evaluation corpus (see Sect. 4). The source-words and their corresponding target-words are highlighted in bold face. The correct translation of the source-noun (e.g. Grenze) as determined by the actual translators is replaced by the set of alternative translations (e.g. \{ border, frontier, boundary, limit, periphery, edge $\}$ ) as proposed by the word-to-word dictionary of Fig. 5 (see Sect. 4).

The problem to be solved is to find a correct translation of the source-word using only minimal contextual information. In our approach,
(ID 160867) Es gibt einige alte Passvorschriften, die besagen, dass man einen Pass haben muss, wenn man die Grenze überschreitet. There are some old provisions regarding passports which state that people crossing the \{border/ frontier/ boundary/ limit/ periphery/ edge $\}$ should have their passport on them.

(ID 201946) Es gibt schliesslich keine Lösung ohne die Mobilisierung der bürgerlichen Gesellschaft und die Solidarität der Demokraten in der ganzen Welt. There can be no solution, finally, unless civilian \{company / society / companionship/ party / associate is mobilized and solidarity demonstrated by democrats throughout the world.

Figure 3: Examples for target-word ambiguities

the decision between alternative target-nouns is done by using only information provided by the governing target-verb. The key idea is to back up this minimal information with the condensed and precise information of a probabilistic classbased lexicon. The criterion for choosing an alternative target-noun is thus the best fit of the lexical and semantic information of the targetnoun to the semantics of the argument-slot of the target-verb. This criterion is checked by a simple lexicon look-up where the target-noun with highest estimated class-based frequency is determined. Formally, choose the target-noun $\hat{n}$ (and a class $\hat{c}$ ) such that

$$
f_{\hat{c}}(\hat{n})=\max _{n \in N, c \in C} f_{c}(n)
$$

where $f_{c}(n)=f(n) p(c \mid n)$ is the estimated frequency of $n$ in the sample of objects of a fixed target-verb, $p(c \mid n)$ is the class-membership probability of $n$ in $c$ as determined by the probabilistic lexicon, and $f(n)$ is the frequency of $n$ in the combined sample of objects and translation alternatives ${ }^{1}$.

Consider example ID 160867 from Fig. 3. The ambiguity to be resolved concerns the direct objects of the verb cross whose lexical entry is partly shown in Fig. 2. Class 19 and the noun border is the pair yielding a higher estimated frequency than any other combination of a class and an alternative translation such as boundary. Similarly, for example ID 301946, the pair of the

\footnotetext{
${ }^{1}$ Note that $p(\hat{c})=\max _{c \in C} p(c)$ in most, but not all cases.
} 
target-noun society and class 6 gives highest estimated frequency of the objects of mobilize.

\section{Evaluation}

We evaluated our resolution methods on a pseudo-disambiguation task similar to that used in Rooth et al. (1999) for evaluating clustering models. We used a test set of $298\left(v, n, n^{\prime}\right)$ triples where $(v, n)$ is chosen randomly from a test corpus of pairs, and $n^{\prime}$ is chosen randomly according to the marginal noun distribution for the test corpus. Precision was calculated as the number of times the disambiguation method decided for the non-random target noun $(\hat{n}=n)$.

As shown in Fig. 4, we obtained $88 \%$ precision for the class-based lexicon (ProbLex), which is a gain of $9 \%$ over the best clustering model and a gain of $15 \%$ over the human baseline $^{2}$.

\begin{tabular}{|c|c|c|c|}
\hline ambiguity & $\begin{array}{c}\text { human } \\
\text { baseline }\end{array}$ & clustering & ProbLex \\
\hline \hline 2 & $73.5 \%$ & $79.0 \%$ & $88.3 \%$ \\
\hline
\end{tabular}

Figure 4: Evaluation on pseudo-disambiguation task for noun-ambiguity

The results of the pseudo-disambiguation could be confirmed in a further evaluation on a large number of randomly selected examples of a real-world bilingual corpus. The corpus consists of sentence-aligned debates of the European parliament $($ mlcc $=$ multilingual corpus for cooperation) with ca. 9 million tokens for German and English. From this corpus we prepared a gold standard as follows. We gathered word-to-word translations from online-available dictionaries and eliminated German nouns for which we could not find at least two English translations in the mlcc-corpus. The resulting 35 word dictionary is shown in Fig. 5. Based on this dictionary, we extracted all bilingual sentence pairs from the corpus which included both the source-noun and the target-noun. We restricted the resulting ca. 10,000 sentence pairs to those which included a source-noun from this

\footnotetext{
${ }^{2}$ Similar results for pseudo-disambiguation were obtained for a simpler approach which avoids another EM application for probabilistic class labeling. Here $\hat{n}$ (and $\hat{c}$ ) was chosen such that $f_{\hat{c}}(v, \hat{n})=$ $\max _{c, n}\left(\left(f_{L C}(v, n)+1\right) p_{L C}(c \mid v, n)\right)$. However, the sensitivity to class-parameters was lost in this approach.
}

dictionary in the object position of a verb. Furthermore, the target-object was required to be included in our dictionary and had to appear in a similar verb-object position as the sourceobject for an acceptable English translation of the German verb. We marked the German noun $n_{g}$ in the source-sentence, its English translation $n_{e}$ as appearing in the corpus, and the English lexical verb $v_{e}$. For the 35 word dictionary of Fig. 5 this semi-automatic procedure resulted in a test corpus of 1,340 examples. The average ambiguity in this test corpus is 8.63 translations per source-word. Furthermore, we took the semantically most distant translations for 25 words which occured with a certain frequency in the evaluation corpus. This gave a corpus of 814 examples with an average ambiguity of 2.83 translations. The entries belonging to this dictionary are highlighted in bold face in Fig. 5. The dictionaries and the related test corpora are available on the web ${ }^{3}$.

We believe that an evaluation on these test corpora is a realistic simulation of the hard task of target-language disambiguation in real-word machine translation. The translation alternatives are selected from online dictionaries, correct translations are determined as the actual translations found in the bilingual corpus, no examples are omitted, the average ambiguity is high, and the translations are often very close to each other. In constrast to this, most other evaluations are based on frequent uses of only two clearly distant senses that were determined as interesting by the experimenters.

Fig. 6 shows the results of lexical ambiguity resolution with probabilistic lexica in comparison to simpler methods. The rows show the results for evaluations on the two corpora with average ambiguity of 8.63 and 2.83 respectively. Column 2 shows the percentage of correct translations found by disambiguation by random choice. Column 3 presents as another baseline disambiguation with the major sense, i.e., always choose the most frequent targetnoun as translation of the source-noun. In column 4 , the empirical distribution of $(v, n)$ pairs in the training corpus extracted from the BNC is used as disambiguator. Note that this method yields good results in terms of precision $(\mathrm{P}=$ \#correct / \#correct + \#incorrect $)$, but is much

\footnotetext{
${ }^{3}$ http: //www.ims.uni-stuttgart.de/projekte/gramotron/
} 


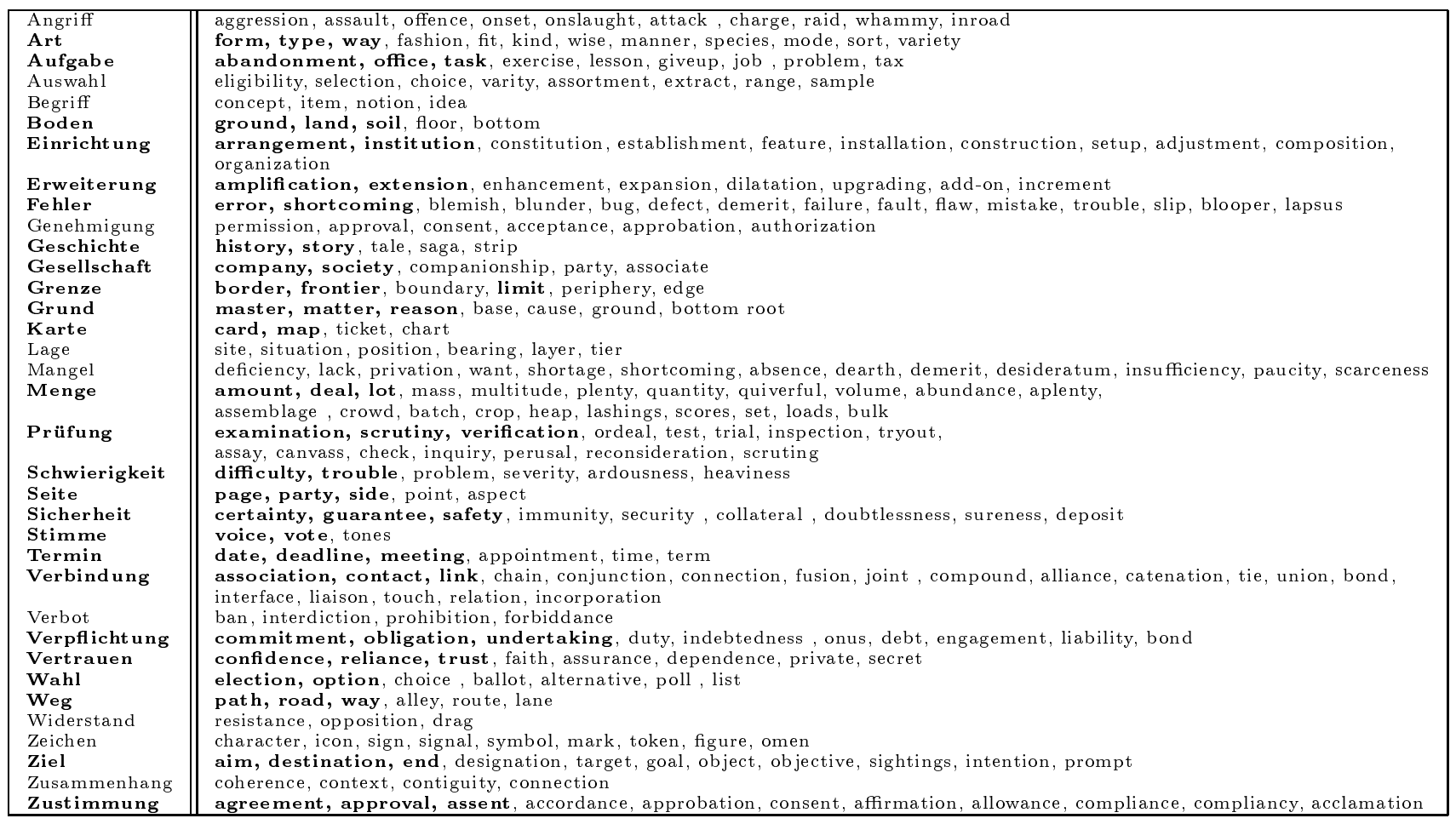

Figure 5: Dictionaries extracted from online resources

\begin{tabular}{|c|c|c|c|c|c|}
\hline ambiguity & random & $\begin{array}{c}\text { major } \\
\text { sense }\end{array}$ & $\begin{array}{c}\text { empirical } \\
\text { distrib. }\end{array}$ & clustering & ProbLex \\
\hline \hline 8.63 & $14.2 \%$ & $31.9 \%$ & $\begin{array}{l}\text { P: } 46.1 \% \\
\text { E: } 36.2 \%\end{array}$ & $43.3 \%$ & $49.4 \%$ \\
& $35.9 \%$ & $45.5 \%$ & $\begin{array}{l}\text { P: } 60.8 \% \\
\text { E: } 49.4 \%\end{array}$ & $61.5 \%$ & $68.2 \%$ \\
\hline
\end{tabular}

Figure 6: Disambiguation results for clustering versus probabilistic lexicon methods

worse in terms of effectiveness $(\mathrm{E}=$ \#correct $/$ \#correct + \#incorrect + \#don't know). The reason for this is that even if the distribution of $(v, n)$ pairs is estimated quite precisely for the pairs in the large training corpus, there are still many pairs which receive the same or no positive probability at all. These effects can be overcome by a clustering approach to disambiguation (column 5). Here the class-smoothed probability of a $(v, n)$ pair is used to decide between alternative target-nouns. Since the clustering model assigns a more fine-grained probability to nearly every pair in its domain, there are no don't know cases for comparable precision values. However, the semantically smoothed probability of the clustering models is still too coarse-grained when compared to a disambigua- tion with a probabilistic lexicon. Here a further gain in precision and equally effectiveness of ca. $7 \%$ is obtained on both corpora (column 6). We conjecture that this gain can be attributed to the combination of frequency information of the nouns and the fine-tuned distribution on the selection classes of the the nominal arguments of the verbs. We believe that including the set of translation alternatives in the ProbLex distribution is important for increasing efficiency, because it gives the disambiguation model the opportunity to choose among unseen alternatives. Furthermore, it seems that the higher precision of ProbLex can not be attributed to filling in zeroes in the empirical distribution. Rather, we speculate that ProbLex intelligently filters the empirical distribution by reducing maximal 
counts for observations which do not fit into classes. This might help in cases where the empirical distribution has equal values for two alternatives.

\begin{tabular}{|c|c|c|c|}
\hline source & target & correct & accept. \\
\hline Seite & $\begin{array}{l}\text { page } \\
\text { side }\end{array}$ & $76.9 \%$ & $76.9 \%$ \\
\hline Sicherheit & $\begin{array}{l}\text { guarantee } \\
\text { safety }\end{array}$ & $93.8 \%$ & $93.0 \%$ \\
\hline Verbindung & $\begin{array}{l}\text { connection } \\
\text { link }\end{array}$ & $58.8 \%$ & $93.8 \%$ \\
\hline Verpflichtung & $\begin{array}{l}\text { commitment } \\
\text { obligation }\end{array}$ & $73.2 \%$ & $94.1 \%$ \\
\hline Ziel & $\begin{array}{l}\text { objective } \\
\text { target }\end{array}$ & $72.5 \%$ & $85.5 \%$ \\
\hline overall precision & & $78 \%$ & $90 \%$ \\
\hline
\end{tabular}

Figure 7: Precision for finding correct and acceptable translations by lexicon look-up

Fig. 7 shows the results for disambiguation with probabilistic lexica for five sample words with two translations each. For this dictionary, a test corpus of 219 sentences was extracted, 200 of which were additionally labeled with acceptable translations. Precision is $78 \%$ for finding correct translations and $90 \%$ for finding acceptable translations.

Furthermore, in a subset of 100 test items with average ambiguity 8.6, a human judge having access only to the English verb and the set of candidates for the target-noun, i.e. the information used by the model, selected among translations. On this set, human precision was $39 \%$.

\section{Discussion}

Fig. 8 shows a comparison of our approach to state-of-the-art unsupervised algorithms for word sense disambiguation. Column 2 shows the number of test examples used to evaluate the various approaches. The range is from ca. 100 examples to ca. 37,000 examples. Our method was evaluated on test corpora of sizes 219,814 , and 1,340. Column 3 gives the average number of senses/translations for the different disambiguation methods. Here the range of the ambiguity rate is from 2 to about 9 senses ${ }^{4}$. Column 4

\footnotetext{
${ }^{4}$ The ambiguity factor 2.27 attributed to Dagan and Itai's (1994) experiment is calculated by dividing their average of 3.27 alternative translations by their average of 1.44 correct translations. Furthermore, we calculated the ambiguity factor 3.51 for Resnik's (1997) experiment
}

shows the random baselines cited for the respective experiments, ranging from ca. $11 \%$ to $50 \%$. Precision values are given in column 5 . In order to compare these results which were computed for different ambiguity factors, we standardized the measures to an evaluation for binary ambiguity. This is achieved by calculating $p^{1 / \log _{2} a m b}$ for precision $p$ and ambiguity factor $a m b$. The consistency of this "binarization" can be seen by a standardization of the different random baselines which yields a value of ca. $50 \%$ for all approaches ${ }^{5}$. The standardized precision of our approach is ca. $79 \%$ on all test corpora. The most direct point of comparison is the method of Dagan and Itai (1994) which gives $91.4 \%$ precision (92.7\% standardized) and $62.1 \%$ effectiveness $(66.8 \%$ standardized) on 103 test examples for target word selection in the transfer of Hebrew to English. However, compensating this high precision measure for the low effectiveness gives values comparable to our results. Dagan and Itai's (1994) method is based on a large variety of grammatical relations for verbal, nominal, and adjectival predicates, but no class-based information or slot-labeling is used. Resnik (1997) presented a disambiguation method which yields $44.3 \%$ precision (63.8\% standardized) for a test set of 88 verb-object tokens. His approach is comparable to ours in terms of informedness of the disambiguator. He also uses a class-based selection measure, but based on WordNet classes. However, the task of his evaluation was to select WordNet-senses for the objects rather than the objects themselves, so the results cannot be compared directly. The same is true for the SEnseval evaluation exercise (Kilgarriff and Rosenzweig, 2000) - there word senses from the HECTOR-dictionary had to be disambiguated. The precision results for the ten unsupervised systems taking part in the competitive evaluation ranged from $20-65 \%$ at efficiency values from $3-54 \%$. The Senseval standard is clearly beaten by the earlier results of Yarowsky (1995) (96.5\% precision) and Schütze (1992) (92\% precision). However, a comparison to these re-

from his random baseline $28.5 \%$ by taking $100 / 28.5$; reversely, Dagan and Itai's (1994) random baseline can be calculated as $100 / 2.27=44.05$. The ambiguity factor for SEnSEVAl is calculated for the noun task in the English Senseval test set.

${ }^{5}$ Note that we are guaranteed to get exactly $50 \%$ standardized random baseline if random $\cdot a m b=100 \%$. 


\begin{tabular}{|c|c|c|c|c|c|c|}
\hline $\begin{array}{l}\text { disambiguation } \\
\text { method }\end{array}$ & $\begin{array}{c}\text { Corpus } \\
\text { size }\end{array}$ & ambiguity & random & precision & $\begin{array}{c}\text { random } \\
\text { (standardized) }\end{array}$ & $\begin{array}{c}\text { precision } \\
\text { (standardized) }\end{array}$ \\
\hline ProbLex & $\begin{array}{c}1340 \\
814 \\
219\end{array}$ & $\begin{array}{c}8.63 \\
2.83 \\
2\end{array}$ & $\begin{array}{l}14.2 \% \\
35.9 \% \\
50.0 \%\end{array}$ & $\begin{array}{l}49.4 \% \\
68.2 \% \\
78.0 \%\end{array}$ & $\begin{array}{l}53.4 \% \\
50.5 \% \\
50.0 \%\end{array}$ & $\begin{array}{l}79.7 \% \\
77.5 \% \\
78.0 \%\end{array}$ \\
\hline Dagan, Itai 94 & 103 & 2.27 & $44.1 \%$ & $\begin{array}{l}\text { P: } 91.4 \% \\
\text { E: } 62.1 \%\end{array}$ & $50.0 \%$ & $\begin{array}{l}\text { P: } 92.7 \% \\
\text { E: } 66.8 \%\end{array}$ \\
\hline Resnik 97 & 88 & 3.51 & $28.5 \%$ & $44.3 \%$ & $50.0 \%$ & $63.8 \%$ \\
\hline SENSEVAL 00 & 2756 & 9.17 & $10.9 \%$ & $\begin{array}{l}\text { P: } 20-65 \% \\
\text { E: } 3-54 \%\end{array}$ & $50.0 \%$ & $\begin{array}{l}\text { P: } 60-87 \% \\
\text { E: } 33-83 \%\end{array}$ \\
\hline $\begin{array}{l}\text { Yarowsky } 95 \\
\text { Schütze } 92\end{array}$ & $\begin{array}{l}\sim 37000 \\
\sim 3000\end{array}$ & $\begin{array}{l}2 \\
2\end{array}$ & $\begin{array}{l}50.0 \% \\
50.0 \%\end{array}$ & $\begin{array}{l}96.5 \% \\
92.0 \%\end{array}$ & $\begin{array}{l}50.0 \% \\
50.0 \%\end{array}$ & $\begin{array}{l}96.5 \% \\
92.0 \%\end{array}$ \\
\hline
\end{tabular}

Figure 8: Comparison of unsupervised lexical disambiguation methods.

sults is again somewhat difficult. Firstly, these approaches were evaluated on words with two clearly distant senses which were determined by the experimenters. In contrast, our method was evalutated on randomly selected actual translations of a large bilingual corpus. Furthermore, these approaches use large amounts of information in terms of linguistic categorizations, large context windows, or even manual intervention such as initial sense seeding (Yarowsky, 1995). Such information is easily obtainable, e.g., in IR applications, but often burdensome to gather or simply unavailable in situations such as incremental parsing or translation.

\section{Conclusion}

The disambiguation method presented in this paper deliberately is restricted to the limited amount of information provided by a probabilistic class-based lexicon. This information yet proves itself accurate enough to yield good empirical results, e.g., in target-language disambiguation. The probabilistic class-based lexica are induced in an unsupervised manner from large unannotated corpora. Once the lexica are constructed, lexical ambiguity resolution can be done by a simple lexicon look-up. In targetword selection, the most frequent target-noun whose semantics fits best to the semantics of the argument-slot of the target-verb is chosen. We evaluated our method on randomly selected examples from real-world bilingual corpora which constitutes a realistic hard task. Disambiguation based on probabilistic lexica performed satisfactory for this task. The lesson learned from our experimental results is that hybrid models combining frequency information and class-based probabilities outperform both pure frequencybased models and pure clustering models. Further improvements are to be expected from extended lexica including, e.g., adjectival and prepositional predicates.

\section{References}

Glenn Carroll and Mats Rooth. 1998. Valence induction with a head-lexicalized PCFG. In Proceedings of EMNLP-3, Granada.

Ido Dagan and Alon Itai. 1994. Word sense disambiguation using a second language monolingual corpus. Computational Linguistics, 20:563-596.

Adam Kilgarriff and Joseph Rosenzweig. 2000. English Senseval: Report and results. In Proceedings of LREC 2000.

Philip Resnik. 1997. Selectional preference and sense disambiguation. In Proceedings of the ANLP'97 Workshop: Tagging Text with Lexical Semantics: Why, What, and How?, Washington, D.C.

Mats Rooth, Stefan Riezler, Detlef Prescher, Glenn Carroll, and Franz Beil. 1999. Inducing a semantically annotated lexicon via EMbased clustering. In Proceedings of the 37th Annual Meeting of the Association for Computational Linguistics (ACL'99), Maryland.

Hinrich Schütze. 1992. Dimensions of meaning. In Proceedings of Supercomputing '92.

David Yarowsky. 1995. Unsupervised word sense disambiguation rivaling supervised methods. In Proceedings of the 33rd Annual Meeting of the Association for Computational Linguistics (ACL'95), Cambridge, MA. 